\title{
L'émergence improbable du domaine et du monde francophones au Portugal entre 1945 et 1970
}

\section{Maria Hermínia Amado Laurel}

\section{(2) OpenEdition \\ 12 Journals}

Édition électronique

URL : https://journals.openedition.org/dhfles/496

DOI : $10.4000 /$ dhfles.496

ISSN : 2221-4038

Éditeur

Société Internationale pour l'Histoire du Français Langue Étrangère ou Seconde

Édition imprimée

Date de publication : 1 janvier 2008

Pagination : $311-339$

ISSN : 0992-7654

\section{Référence électronique}

Maria Hermínia Amado Laurel, « L'émergence improbable du domaine et du monde francophones au

Portugal entre 1945 et 1970 », Documents pour l'histoire du français langue étrangère ou seconde [En ligne], 40/41 | 2008, mis en ligne le 11 janvier 2011, consulté le 27 mai 2021. URL : http://

journals.openedition.org/dhfles/496 ; DOl : https://doi.org/10.4000/dhfles.496

Ce document a été généré automatiquement le 27 mai 2021.

(c) SIHFLES 


\title{
L'émergence improbable du domaine et du monde francophones au Portugal entre 1945 et 1970
}

\author{
Maria Hermínia Amado Laurel
}

1 Étudier l'émergence du domaine et du monde francophones dans les études françaises au Portugal entre 1945 et 1970 requiert une attention particulière sur une période singulièrement importante de l'histoire de cet enseignement, dans un pays qui renonçait volontairement, à l'époque, à suivre le cours de l'histoire européenne, et qui employait toutes ses ressources matérielles et spirituelles à la construction d'une histoire idéalisée, voire instrumentée. Effectivement, cette histoire, ses mentors la voulaient unique, sans égale, de par la supériorité des valeurs qu'elle avait la mission de divulguer. Le caractère sacré de cette mission, qui la consacrait, selon eux, dans l'histoire des civilisations mondiales, justifiait en lui-même sa continuité ad aeternam ${ }^{1}$.

2 L'évolution historique du Portugal ne peut être abordée sans que l'on tienne compte d'un problème de fond: la confrontation entre les différents courants idéologiques autour de la question des territoires non européens sous administration portugaise. Ce problème devient incontournable pour la politique intérieure et extérieure du pays ; il a déterminé les deux changements de régime politique majeurs que le pays a connus au $\mathrm{XX}^{\mathrm{e}}$ siècle. Suite en partie à l'Ultimatum anglais de $1890^{2}$, le régime monarchique en vigueur depuis la naissance du pays au XII ${ }^{\text {e }}$ siècle, succombe devant le régime républicain en 1910 ; suite au soulèvement militaire du 25 avril 1974 et à la chute du régime "Estado Novo", dont la Constitution de 1933 consacre les principes, l'indépendance est accordée, de manière abrupte, à tous les territoires sous administration portugaise, de la côte africaine à Timor, en Océanie (à l'exception des archipels de Madère et des Açores, qui acquièrent le statut de "régions autonomes »). Le Portugal se retrouve confronté à la proximité européenne de ses frontières péninsulaires et devient membre de la Communauté en 1986.

3 Cette étude nous amènera par voie de conséquence à fonder notre analyse sur la connaissance $\mathrm{du}$ fond historique, politique, socioculturel et institutionnel portugais 
dans lequel évoluent les études françaises au long de la période en question, tout en tenant compte, également, des lignes de divergence qui caractérisaient la situation portugaise face à la situation internationale; la mise en lumière de ces lignes de divergence nous aidera à mieux comprendre les raisons de l'émergence tardive du domaine et du monde francophones dans les études françaises au Portugal.

Les études françaises ${ }^{3}$ étaient centrées au Portugal, tout particulièrement pendant les années 1945-1970, presque exclusivement sur les études franco-françaises, bien que celles-ci incluent des auteurs belges ou suisses de langue française, globalement identifiés comme auteurs français. Plusieurs facteurs expliquent la permanence de ce choix ${ }^{4}$ pendant la période que nous considérons, et bien au-delà ${ }^{5}$.

5 Si les origines de ce choix remontent à l'attrait qu'exerce la culture française sur l'histoire culturelle portugaise, les raisons pour lesquelles cette situation se prolonge pendant les années 1945-1970 nous semblent dépasser ce cadre référentiel. Leur étude constitue un champ d'analyse privilégié, dans lequel les pratiques d'enseignement et les options politiques et idéologiques du régime se rejoignent. Effectivement, l'étude de ces circonstances met en lumière le décalage profond qui existait, à l'époque, entre les pratiques institutionnelles d'enseignement et la situation réelle de leur objet d'étude : les études françaises restent centrées sur certaines images de la France, choisies en fonction de l'orientation idéologique des études de l'enseignement officiel; elles excluent ou éludent une nouvelle réalité : celle de l'émergence des francophonies, qui conféreront une dimension différente et singulière aux études françaises, tout en accentuant leur décentrement face à la « métropole » parisienne ${ }^{6}$. Les années 1945-1970 marquent le tournant décisif non seulement dans l'histoire européenne et coloniale, mais aussi dans l'histoire des études françaises elles-mêmes, en conséquence d'une plus grande divulgation de la production non hexagonale ${ }^{7}$ (Moura, J.-M. 1999).

6 Effectivement, cette période correspond aux années où sont publiés les principaux textes d'affirmation de la conscience anticoloniale, période politiquement engagée par excellence ${ }^{8}$. La fin des années soixante-dix s'ouvre, par contre, à d'autres raisons d'être de la production littéraire francophone, d'où émerge, à son tour, la constitution de champs littéraires individualisés (Moura, J.-M. 1999 : 147)9.

7 La période comprise entre 1945 et 1970 situe l'émergence du domaine et du monde francophones dans les pays colonisés par la France, mais aussi en France, dont le rapport aux colonies est dicté par les nouveaux enjeux politiques internes et externes qui se posent au pays en conséquence de sa participation à la Seconde Guerre mondiale. Si nous rapportons cette période au contexte portugais, nous reconnaîtrons aisément qu'elle renvoie à une situation pour laquelle ce que nous nommons aujourd'hui, en termes comparatistes, les "espaces francophones ", était loin d'intégrer l"espace' des représentations symboliques du français véhiculées par les études françaises.

Si l'on considère ces dates dans l'histoire de l'enseignement secondaire ou universitaire au Portugal, nous sommes forcés d'admettre qu'elles renvoient à la réalité institutionnelle, idéologique et politique d'un régime et d'un gouvernement pour lesquels la notion d'« espaces francophones » ne pouvait pas faire, à l'époque, grand sens. Ce qui ne doit pourtant pas faire croire que les études françaises étaient absentes de notre système d'enseignement à l'époque. Bien au contraire, ces études y étaient présentes dès la fondation des lycées en $1836^{10}$, et avant cela, dans l'enseignement privé $^{11}$, par l'initiative de maîtres de langues, de "demoiselles » (françaises, suisses, belges, etc.) et d'autres instances qui travaillaient dans l'enseignement laïc, religieux, 
ou militaire ${ }^{12}$. La non-viabilité de l'attribution de sens à l'expression en cause dans le contexte d'enseignement du français au Portugal à l'époque s'explique du fait que la réalité sous-jacente à cette expression - domaine et monde francophones - est le fruit d'un processus historique européen et colonial différent pour les deux pays. Cette expression était tout simplement inconciliable avec la vision du monde de la « lusitanéité » bâtie par le régime.

9 Un paragraphe s'impose ici, pour nous aider à comprendre le changement de cap idéologique du régime "Estado Novo » survenu à partir des années cinquante du $\mathrm{XX}^{\mathrm{e}}$ siècle. Un changement de nature régressive, et qu'il faudra considérer selon une double perspective. D'une part, par rapport à l'évolution même des points de vue internes sur la question coloniale, ce que la confrontation des désignations pour ces territoires selon les différents régimes politiques qui se sont succédé à partir de l'Ultimatum anglais de 1890 et au long du XXe siècle, aidera facilement à comprendre (I). D'autre part, par notre prise en compte de l'évolution de la question «francophone » entre 1945 et les années soixante et soixante-dix, années des indépendances et de fin d'un cycle (II).

(I) Les désignations de "province » ou de "colonie» recouvrent des points de vue difficilement conciliables face à la question coloniale. Si l'origine du concept de " province africaine», hérité de la pensée française de 1789, détermine des courants qui défendent, au long du XIX siècle, des politiques d'«assimilation» (le principe de l'égalité entre tous les hommes étant sauvegardé par des modèles politiques et juridiques reconnus dans les métropoles) et la "centralisation » (autour d'un siège du pouvoir unique, pour la sauvegarde des décisions contre des intérêts locaux), celle du concept de "colonie", par contre, nous fait remonter aux tendances politiques affichées par la République portugaise dans son texte constitutionnel de 1911. Le changement de nom du ministère de la Marine et de l'Outre-mer en celui de ministère de la Marine et des Colonies, légitimé par ce texte, se révèle significatif dans ce contexte. Le rapport de dépendance, voire de soumission, unilatérale, institué par le tandem «métropole/colonie » - dont le champ sémantique recouvre les connotations que les études postcoloniales se proposent d'étudier -, présupposait, à l'opposé, pour la pensée républicaine la possibilité de l'émancipation, et visait l'acquisition progressive par les territoires colonisés de l'autonomie face à la métropole. Les expressions "province » et "colonie » renvoient ainsi à des positions idéologiques et politiques antagonistes, celles qui opposaient, au début du siècle, monarchistes et républicains. La poursuite de la reconnaissance internationale du régime colonial portugais conduira à la participation désastreuse du pays à la PremièreGuerre mondiale; elle conduira également à la rédaction de l'Acte colonial en 1930. Celui-ci reconnaissait clairement la nécessité d'une séparation entre les modèles politiques et juridiques métropolitains et ceux adaptés aux réalités locales, dans la recherche de la meilleure solution face à la découverte d'us, coutumes et pratiques inconciliables avec les modèles européens (Caetano M. 1974: 17-24). L'histoire a prouvé l'iniquité du statut de citoyenneté, différent pour les «indigènes", les «assimilés" et les "citoyens ». Or, très curieusement, c'est vers les vieilles idées monarchistes d' «assimilation» et de "centralisme", que semblent se tourner les choix politiques portugais à partir des années cinquante, réunis désormais sous le label de l'« intégration » (Caetano M. 1974 : 24).

11 (II) L'attribution, en 1921, du prix Goncourt ${ }^{13}$ à l'écrivain antillais René Maran, pour Batouala: véritable roman nègre, a constitué sans doute l'un des premiers signes de 
légitimation d'un champ littéraire nouveau et prometteur: celui de la littérature francophone. Les retentissements du lancement simultané à Paris et à Dakar, en 1947, par A. Diop, Aimé Césaire et Léopold S. Senghor de la revue Présence africaine,confirmeront ce nouveau champ, presque trente ans révolus après l'attribution de ce prix à R. Maran, dont, par ailleurs, ils mettront en lumière, le caractère circonstanciel. Une revue qui fut pourtant précédée, il convient de ne pas l'oublier, de la publication, en 1934, de L'Étudiant noir (autre revue) par le martiniquais Aimé Césaire et le guyanais L. G. Damas. C'est dans cette feuille, fortement engagée en faveur des indépendances, que fut employée pour la première fois l'expression "négritude ${ }^{14}$, celle-ci traduisant, en même temps qu'un sentiment d'appartenance différentielle (jugé plus tard "raciste» par A. Césaire), le désir provocateur d'émancipation culturelle et d'indépendance politique. Des événements tels que le "Congrès des écrivains et artistes noirs » à la Sorbonne, en 1956, organisé par A. Diop et le premier «Festival mondial des arts nègres » en 1966, à Dakar, dans un Sénégal récemment devenu indépendant, par le même A. Diop et Léopold S. Senghor ${ }^{15}$, et le constat de la situation portugaise, suffiraient à nous faire comprendre l'écart affiché par le gouvernement portugais face à la question des indépendances, pendant les mêmes années.

Considérant que l'histoire de l'enseignement des langues et des littératures est profondément conditionnée par l'environnement politique des pays où ces enseignements ont lieu, et par les valeurs qui y sont sous-jacentes, il est aisé de comprendre que le contexte où les études françaises ont évolué au Portugal entre 1945 et 1970 s'écartait profondément de celui qui déterminait la situation française, favorable à l'émergence du « domaine et du monde francophones ». D'après la situation politique et les repères historiques que nous venons d'évoquer à larges traits, il devient clair que la notion d'études francophones ne pouvait intégrer le vocabulaire officiel de l'enseignement portugais.

D'autres limites chronologiques doivent aussi être considérées pour mettre en perspective cette émergence au Portugal, principalement celles, assez larges, qui balisent l'« Estado Novo » entre 1933 et 1974, dans un pays, replié sur lui-même, qui avait gardé le statut de neutralité pendant le second conflit mondial et qui avait prolongé son identité coloniale jusqu'en 1974.

Trois axes d'approche s'avèrent importants à ce moment de notre réflexion sur cette période, que nous considérerons pourtant avec plus d'attention à partir de 1945 :

1. La mise en parallèle de la situation politique portugaise et internationale.

2. La référence au champ éditorial francophone et lusophone, au niveau de quelques publications et traductions.

3. La contextualisation des études françaises au niveau de l'enseignement secondaire et universitaire.

15 Ces quelques axes de réflexion nous permettront de comprendre, d'autre part, l'interdépendance étroite, dans le cas portugais, entre l'émergence des francophonies et celle des lusophonies postcoloniales, les deux domaines ayant subi des processus d'affirmation parallèles dans le contexte politique tardif de ce pays, face à la question coloniale européenne. 


\section{La situation portugaise et la situation internationale, notamment française, à partir de l'après-guerre}

Afin de bien mesurer l'écart temporel que le processus des autonomies, voire des indépendances coloniales, a connu en France et au Portugal, il nous semble important de rappeler quelques dates et quelques événements :

\section{5 :}

Domaine international :

- Création de l'ONU, l'Organisation des Nations Unies; le Portugal, après le refus à sa demande d'adhésion, imposé en 1946, y sera admis le 15 décembre 1955.

Portugal :

1. - Fondation du MUD, Movimento de União Democrática (Mouvement d'union démocratique), par des hommes politiques, des intellectuels et des artistes de l'opposition à gauche : Mário Soares, Salgado Zenha, Octávio Pato, Júlio Pomar, Mário Sacramento.

2. - Institution d'une nouvelle force policière, la «Police internationale pour la défense de l'État » (PIDE), dont l'action était censée être « autonome » par rapport à celle exercée par le pouvoir judiciaire ${ }^{16}$.Après 1945 , au moment où l'Europe célébrait la liberté, le régime de Salazar se verra contesté à droite et à gauch $\mathrm{e}^{17}$. Pour lutter contre les oppositions le régime se durcit, et se renferme davantage sur lui-même.

3. - Organisation de la «Conférence inter-académique pour l'unification orthographique de la langue portugaise», pour la sauvegarde de «l'unité universelle» / «unité intercontinentale » de cette langue.

L'histoire de l'accord orthographique dont le propos était d'unifier la langue portugaise ou, du moins, d'en estomper les diverses variantes, nous aide à comprendre la situation du Portugal face aux autres pays, «colonies» ou "provinces» sous administration portugaise ${ }^{18}$. Cette histoire, qui remonte aux débuts du régime républicain ${ }^{19}$, aboutira à la ratification unilatérale de l'accord par le Portugal en 1945. La coïncidence historique entre cette occurrence - l'immédiat après-guerre - et le fait qu'un seul pays, le Brésil, ait été impliqué, jusqu’à la fin du régime " Estado Novo », dans les études linguistiques menant au rapprochement des langues portugaises parlées dans les deux pays pour une orthographe commune, nous semble assez révélatrice des difficultés non seulement de nature linguistique, mais aussi d'ordre politique et surtout identitaire qu'un accord de ce type implique, pour un régime politique fondé sur la conception ambiguë d'une langue qui se voulait d'abord «nationale ", « matricielle », tout en répondant à un désir d'« universalité », puisqu'elle était parlée, dans divers pays, sur plusieurs continents ${ }^{20}$.

L'histoire de cet accord nous montre à quel point la politique de la langue nationale suivie par un pays peut avoir d'incidences sur la politique des langues étrangères et des relations internationales qu'il défend. Sous-jacente à cette histoire, on retrouve implicite, la question coloniale dans toute son amplitude, fondée sur le rapport à une langue qui s'est voulue "nationale » et unifiée, tout autant qu' " universelle»; mais aussi la question coloniale dans ce qu'elle impliquait pour chaque cas spécifique : tout d'abord, les rapports avec le Brésil, et, à partir de $1986^{21}$, avec les anciennes colonies ${ }^{22}$.

L'option franco-française dans l'histoire de l'enseignement du français au Portugal nous semble ainsi s'accorder entièrement, en toute cohérence, avec l'orientation de la politique linguistique concernant la langue nationale. Langue nationale à la portée universelle, la langue portugaise remplissait le rôle joué par d'autres langues 
européennes, véhicules d'expression d'autres peuples, elles aussi chargées de diffuser un modèle de civilisation. Mission que la langue française s'était attribuée depuis longtemps, dans l'histoire de la civilisation occidentale ${ }^{23}$.

Ce qui explique d'autre part, le fait, surprenant au premier abord, mais absolument logique dans le fond, que l'enseignement d'une langue étrangère telle que le français, fût conçu, selon les programmes d'enseignement et les manuels d'enseignement utilisés au Portugal, au service des objectifs de l'idéologie nationale de l'époque qui nous concerne, davantage que pour des propos de véritable entente et de partage de valeurs entre les utilisateurs de cette langue en dehors de tout contexte nationaliste ${ }^{24}$.

Situation pourtant paradoxale, si nous considérons que c'est justement la période pendant laquelle émerge la notion de francophonie en tant que synonyme d'émancipation coloniale, et que se fait entendre la voix de ses principaux idéologues. À la notion de francophonie ne pouvait donc pas correspondre, à l'époque, celle de lusophonie, telle que nous la concevons de nos jours, dans une perspective supranationale et supra-géographique, comme l'ensemble des peuples et des pays, indépendants, qui font usage des deux langues, mais une vision du monde proche de celle sous-jacente au concept d'un luso-tropicalisme fortement luso-centré25, défenseur de la suprématie, en exclusivité, de l'aire culturelle nationale.

Revenons à la liste des moments forts qui marquent le décalage de la situation portugaise par rapport à la situation internationale, en particulier par rapport à la situation française, dans l'immédiat après-guerre :

\section{France :}

1. - 1945 : suppression du « statut de l'indigène ».

2. - 1946: abolition de l'expression «Empire colonial français»; création de «l'Union française »; institution des départements et territoires d'outre-mer, respectivement, les DOM, et les TOM.

3. - Années cinquante : le concept de «négritude » d'Aimé Césaire trouve son équivalent sous la plume du poète angolais Agostinho Neto, en celui de « angolanidade » (« angolanité »).

1953 :

1.-Construction d'une nouvelle image du pays avec institution d'une loi qui abolit la désignation d'" Empire colonial portugais » et la remplace par celle de " Provinces d'outremer", tout en gardant théoriquement le même statut administratif pour l'Algarve, l'Alentejo et la Guinée, l'Angola, et les autres territoires d'outre-mer.

31 1954:

1. - Institution de la loi sur le «Statut des indigènes portugais dans les provinces de Guinée, Angola et Mozambique » (abolie en 1961). 
2. - Formation de l'UPNA, l'Union des populations du nord de l'Angola (devenue l'UPA, responsable des premiers massacres des colons et des populations locales en 1961).

3. - Rupture des rapports diplomatiques entre le Portugal et l'Inde.

4. - Restructuration de la PIDE, et création de l'encadrement institu-tionnel de cette police pour les îles atlantiques et pour l'outre-mer ${ }^{27}$.

France :

1. - 1954 : début de la guerre d'Algérie.

2. - 1956 : l'indépendance de la Tunisie et du Maroc est accordée par la France.

En 1960, l'ONU introduit dans sa charte la résolution no 1514 , qui interdit toute pratique coloniale. Le Portugal, de plus en plus isolé, est attaqué à l'ONU par les puissances amies et ennemies. C'est le moment où le régime accentue l'image de son isolement sur la scène internationale, isolement consacré par l'expression de Salazar qui assume la destinée du pays et des Portugais désormais « orgueilleusement seuls » ${ }^{28}$.

Portugal :

1. - 1961 : massacres de colons blancs et de la population indigène noire associée, au Nord de l'Angola. Début de la guerre coloniale dans ce territoire. Annexion des possessions portugaises en Inde, par le gouvernement local ${ }^{29}$.

2. - 1962, 1963 : fondation des universités de l'Angola (Luanda) et du Mozambique (Lourenço Marques).

3. - 1964 : début de la guerre au Mozambique.

4. - 1968 : mort de Salazar ; manifestations de catholiques contre la guerre, à Lisbonne.

5. - 1969 : crises académiques à l'université de Lisbonne et à celle de Coimbra.

6. -1968-1974 : Marcello Caetano est nommé chef du Gouvernement par le Président de la République, l'amiral Américo Thomaz.

7. - 1974 : fin de l'« Estado Novo »; reconnaissance immédiate des Indépendances.

\section{Le champ éditorial national et international}

La situation éditoriale lusophone de l'époque est marquée par des publications qui ont trois origines dont le poids n'est naturellement pas de la même importance, mais dont il convient de tenir compte : aux éditions publiées à Lisbonne, il faudrait ajouter celles qui provenaient de l'Afrique lusophone, et aussi celles produites en URSS, source de beaucoup de publications clandestines et d'endoctrinement idéologique de dissidents mais aussi de jeunes Africains, envoyés par l'opposition au régime portugais, en association avec le parti communiste, dans les universités soviétiques ${ }^{30}$.

Des auteurs africains d'expression portugaise sont édités localement, la presse africaine lusophone ayant commencé son activité dès le milieu du XIX siècle et l'ayant poursuivie avec régularité jusqu'aux années 1950. Des publications périodiques, dont 0 Mensário Administrativo (Luanda, 1947-1951); le Boletim Cultural da Guiné Portuguesa (Bissau, 1946) ; les revues angolaises A Aurora (1856), Cultura (1945-1951) et Mensagem (1949-1965), le Jornal de Angola (1953-1965), méritent une référence. D’autre part, on peut considérer que des liens d'intertextualité africaine étaient présents depuis longtemps dans la littérature lusitanienne, et ils se firent sensibles à partir du Romantisme portugais en particulier ; les travaux d'ethnologie de Teófilo Braga, Adolfo Coelho, Leite de Vasconcelos, de même que leurs recherches sur la langue et la 
littérature orale de langue portugaise ont marqué les premières décennies du $\mathrm{XX}^{\mathrm{e}}$ siècle.

La période de la guerre coloniale a naturellement mis fin à beaucoup d'initiatives éditoriales, la censure ayant rempli un rôle majeur pendant cette période dans les territoires d'outre-mer, indépendamment de l'ampleur du conflit dans chacun d'eux.

L'année 1964 est marquée par quelques événements significatifs du durcissement du régime face à l'intellectualité locale dont, parmi bien d'autres, la fermeture de la résidence des étudiants en provenance des colonies (Casa dos Estudantes do Império) à Lisbonne, l'interdiction de l'éditeur angolais Imbondeiro (fondateur de la collection "Auteurs d'outre-mer », en activité entre 1961-1964), et celle de la Société portu-gaise des écrivains, à Lisbonne (Sociedade Portuguesa de Escritores), à la suite de l'attribution par celle-ci du Grand prix de la nouvelle (Grande Prémio da Novelística) à Luuanda, de Luandino Vieira, à l'époque prisonnier à l'île de Tarrafal, au Cap Vert, dont l'archipel fut épargné par les conflits.

Des traductions portugaises d'Aimé Césaire, Senghor, Ahmadou Kourouma, Mongo Beti, Albert Memmi, surgissent à Lisbonne et Porto dès le début des années 1970, ce qui constitue sans doute une marque de l'ouverture du régime. Ouverture qui répondait au grand espoir collectif de liberté, à la suite de la disparition de Salazar en 1968, et à l'avènement du gouvernement de Marcello Caetano ${ }^{31}$.

En 1974-75, Manuel Ferreira, auteur, avec Gerald Moser, d'une anthologie d'auteurs africains d'expression portugaise ${ }^{32}$, fonde les études de littérature africaine en langue portugaise à la faculté des lettres de l'université de Lisbonne. Cette initiative marque l'institutionnalisation des études lusophones à l'université portugaise.

\section{Le champ des études françaises}

41 Nous pouvons donc constater que la période qui fait suite à la Libération de l'Europe, et en particulier de la France, du joug nazi en 1945 et qui signifie « liberté », correspond, au Portugal, à des années où le durcissement du régime se poursuit, toujours à l'abri de la Constitution de la République portugaise de 1933, cette même constitution qui annonçait, d'après la « révolution nationale » que le régime se proposait d'amener aux Portugais le meilleur des mondes.

Le meilleur des mondes fondé sur la réforme de l'enseignement de 1936, le grand texte idéologique du régime en matière d'éducation ${ }^{33}$. Un régime qui rappelle parfois celui qui régissait l'univers fictionnel du roman d'Aldous Huxley, Brave new world, (1932) ${ }^{34}$, quant aux conséquences d'ordre éthique du projet monstrueux du clonage (ou endoctrinement) des individus, soumis à une idéologie (doctrine) unique, c'est-à-dire, celle du régime de l'« Estado Novo ».

43 L'histoire de la notion de « village global » (qui allait devenir le WWW, en 1991) date des années 1962. À contre-courant de cette histoire, l'enseignement était orienté au Portugal, y compris celui des langues vivantes, par le modèle du « livre unique ", et ceci depuis les années trente jusqu'aux années soixante-dix, avec des périodes de plus ou moins grande fidélité, ce qui est tout aussi vrai.

44 Le désir d'assouplissement du régime finissant transparaît dans quelques textes législatifs concernant l'enseignement, promulgués par le ministre de l'éducation Veiga Simão dès 1970, ministre qui avait été nommé premier président de l'université de 
Lourenço Marques, en 1963, au Mozambique. Son nom restera lié également à l'octroi de l'amnistie aux étudiants arrêtés à la suite de la crise académique survenue à Coimbra en 1969.

Les changements qui se font alors sentir au Portugal, et dont le domaine de l'enseignement est particulièrement révélateur, visent un objectif double: la conciliation du désir d'ouverture au monde du pays avec la sauvegarde de l'unité nationale dans les termes où nous l'avons décrite. Les suites du 25 avril 1974 se chargeront bientôt d'œuvrer à l'impossibilité de son accomplissement.

Effectivement, la "réforme Veiga Simão » (1973) se fonde sur la défense des valeurs essentielles au régime : l'amour de la patrie, la famille, la religion, la morale et la doctrine chrétiennes, traditionnelles dans le pays ; le renforcement de la solidarité des communautés lusitaniennes. Pourtant le discours officiel qui l'institue en loi du pays, change par rapport au texte de 1936: il ne célébrera plus les gloires coloniales; il n'affirmera plus que l'Acte colonial est la charte du Portugal devant les États du monde. Il y sera plutôt question de proposer un modèle éducatif visant à atteindre un autre objectif : celui de la "participation effective à la vie internationale» (loi 5/73). La parution de l'hebdomadaire Expresso en 1970, un des organes les plus importants de la presse d'opinion, dont l'influence est toujours aussi grande de nos jours, semble parfaitement justifiée dans ce contexte.

Je soutenais, au début de mon étude, que les études françaises étaient à l'époque fortement franco-centrées au Portugal. Le lecteur peut donc se demander à quoi ressemblait cette France qu'on admirait tant sous les années dictatoriales au Portugal.

L'une des institutions d'enseignement qui travaillait en étroite collaboration avec le pouvoir français représenté au Portugal, était, à l'époque, l'Alliance française, dont la première délégation venait d'être fondée en 1945 à Lisbonne, et qui devait élargir son réseau à tout le territoire jusqu'en 1974. Cette institution avait pourtant été précédée par l'ouverture de l'Institut français à Lisbonne en 1937, à la suite de la fondation de son prédécesseur à Madrid; le deuxième Institut français du pays, situé dans la ville de Porto, devait développer son action culturelle et sa formation linguistique entre 1942 et 2004.

49 L'idéal de l'apprentissage du français dans ces deux écoles se fondait sur une conviction profondément ancrée dans l'imaginaire collectif de l'époque, selon laquelle posséder cette langue était la marque et la reconnaissance des gens civilisés, cultivés, bien éduqués. La popularité des études françaises à l'époque était une démonstration de la francophilie qui caractérisait la culture portugaise; elle constituait l'expression majeure de la « haute culture » de la civilisation européenne et soutenait, tout en le renforçant, le sentiment de l'appartenance à une communauté civilisationnelle imaginaire où les études françaises faisaient autorité.

50 Les études françaises restaient ainsi, dans le système éducatif national, toujours centrées autour de l'idée de nation et de la mission civilisatrice de la France, dans l'héritage de la pensée républicaine de Jules Ferry, mais dans l'ignorance des cultures coloniales et postcoloniales dans lesquelles la France était déjà bien et bel engagée.

51 Nous retrouvons cette conviction à la base des manuels de langue, où l'enseignement se fonde sur la connaissance de la civilisation française. L'exemple canonique en fut le manuel de Gaston Mauger, Cours de langue et civilisation françaises, le fameux « Mauger 
bleu » adopté pendant de longues années à l'Alliance française, tel qu'il était familièrement appelé.

La forte popularité du français et de la France sous les années de la dictature est encore attestée par la circulation de beaucoup de revues et de journaux français. La lecture du journal Le Monde, des revues féminines ou de mode Elle ou Marie Claire, de la revue aux contenus culturels et d'opinion Réalités, la fréquence de films français au cinéma, la divulgation de la musique française et le succès de la revue Salut les copains auprès des jeunes, constituaient des marques de francophilie évidentes. Pour les uns, ce modèle évoquait l'image d'une France contestataire, libertaire, engagée. Une admiration sans doute teintée d'idéalisme romantique, dont l'origine remonterait à celle ressentie lors de la période libérale au XIX ${ }^{e}$ siècle, récupérant, en quelque sorte l'idéal libertaire révolutionnaire. Pour d'autres, la France devenait le modèle inspirateur de la "démocratie forte " à laquelle aspirait le MUD, mouvement regroupant l'opposition au régime du «Estado Novo » et la revue Vértice, organe de la gauche intellectuelle, dont le premier numéro date de mai 1942.

En tout cas, c'était bien à une image mythifiée de la France que renvoyaient ces représentations, éducatives, politiques, civilisationnelles. À première vue, on pourrait penser que les intellectuels portugais étaient en fin de compte, très orthodoxes vis-àvis de ce goût strictement français, et prisaient un eurocentrisme symbolisé par la France. La preuve en est que les auteurs francophones ne font pas l'objet d'articles dans les revues telles que Vértice. Blaise Cendrars, par exemple, présenté comme un auteur français, y est mentionné comme le principal introducteur de l'écrivain portugais Ferreira de Castro en France, qu'il avait traduit en 1938. Pas de référence pourtant au fait que c'est au Brésil qu'ils se sont connus.

Dans le domaine de l'enseignement, cette admiration était portée sur les valeurs, que les manuels utilisés pour l'enseignement du français au lycée tournaient pourtant au profit des valeurs de l'« Estado Novo ». Le titre de l'un de ces manuels - Regardons vers le pays de France (années 1960-1970) suffirait à l'illustrer.

L'apprentissage du français comme une langue vivante répond donc à l'époque à l'intériorisation d'un modèle de civilisation essentialiste, que la circulation de la presse française (qui était, malgré tout, assez librement consentie par le régime) risquait pourtant, à la longue, d'ouvrir vers d'autres réalités, comme celle de la Francophonie justement.

57 L'admiration pour le modèle français est encore constatée, au niveau universitaire, pendant la période, par la réforme des facultés des Lettres en 1957, qui introduit une discipline nouvelle - la théorie de la littérature. Si la première édition de l'ouvrage du théoricien portugais Aguiar e Silva, Teoria da literatura,en 1967 (Théorie de la littérature, ouvrage à grand succès à l'université), reste proche de l'original de Austin Warren et de René Wellek (1949), la pensée structuraliste, de souche française, séduira les universitaires portugais; la popularité des nouveaux romanciers, dont les œuvres intègrent les programmes universitaires dans les années 1970, devient compréhensible, eux-mêmes étant fortement engagés dans la réflexion métalittéraire.

La réforme de 1957, qui introduit la nouvelle discipline ${ }^{35}$, devait poser les bases du changement de perspective dans les études littéraires, exigé par «le mouvement de restauration nationale entrepris depuis des années " par le régime de l'" Estado Novo ». Ce qui est curieux, c'est que l'introduction de la théorie, si elle n'a pas contribué à 
modifier aussitôt les pratiques pédagogiques, ni les canons des auteurs au programme, ni l'image d'une "certaine idée de la France ", qu'elle a plutôt renforcée à l'étranger, elle allait jouer, à long terme, contre l'idéal éducatif du régime. Celui-ci misait avant tout sur la transmission des valeurs à partir des textes littéraires; or la théorie proposait des concepts, que la critique littéraire devait rendre "opératoires ", dans les méthodologies d'approche des textes qu'elle allait mettre sur pied. La popularité de la discipline parmi les enseignants avait atteint le niveau le plus élevé dans l'exploitation des 'vertus' pédagogiques de l'analyse des textes proposées par les méthodes structuralistes, dans le cadre desquels ont joué un rôle majeur les modèles d'analyse du récit de Gérard Genette ou le modèle sémiotique de Greimas. Outre la publication de Teoria da literatura à laquelle nous venons de faire référence, par un universitaire de Coimbra, seront aussi publiés à Coimbra - alma mater de l'université portugaise -, la Teoria da Linguagem (Théorie du langage), de José Herculano de Carvalho, en 1970 (dont la rédaction commence pourtant bien avant, l'année scolaire de 1959-1960), le livre de Carlos Reis, Técnicas de Análise literária (Techniques pour l'analyse littéraire, de 1978, ouvrage qui connut une grande diffusion à l'univer-sité et au lycée).

Une question importante mériterait d'être soulevée ici : le changement de perspective de l'approche philologique à l'approche théorique a-t-il été propice à l'émergence de la francophonie?

S'il est vrai que la théorie, par ses caractéristiques interdisciplinaires, a bouleversé l'édifice solide du savoir historico-philologico-positiviste, il faut tout de même considérer que, dans la pratique immédiate des enseignements littéraires, la méthode post-lansonienne a perduré dans la pratique de bien des enseignements littéraires, qui restent centrés sur les études franco-françaises. Ce changement a été plutôt favorable, des années plus tard, au renforcement des rapports d'admiration envers la France et surtout ses « maîtres à penser » de la " pensée 68 », le groupe de « la mort de l'auteur ", dont Roland Barthes et Michel Foucault. L'université portugaise, à l'époque, reste une institution profondément centralisée, uniforme, à la hiérarchie rigide, cultivant l'isolement de la vie sociale et le corporatisme, et les études françaises restent fortement rattachées aux études portugaises, dont elles constituent la référence privilégiée, dans le contexte des études de philologie romane ${ }^{36}$.

De toute façon, c'est au niveau de l'enseignement secondaire que la dépendance du français a été plus sensible face à l'enseignement du portugais et des valeurs que celuici était censé transmettre. Le choix des textes, des auteurs au programme, l'orientation de la lecture, les modes de lecture et leur objectif, l'illustrent.

Les représentations de la France étaient véhiculées, à ce niveau, par la lecture des textes littéraires (dans l'original ou en adaptation) qui appuyaient l'enseignement de la langue française. Or, il est très intéressant de vérifier à quel point l'enseignement d'une langue étrangère (en ce cas particulier, le français) constituait encore une occasion de "propagande ", c'était bien le cas, de l'idéologie du régime. J'ai pu analyser, lors d'un colloque de l'APHELLE ${ }^{37}$, la proximité entre le discours juridique national sur le statut de la femme, et l'enseignement du français pour les jeunes lycéennes dans les années 1960 au Portugal. Outre la séparation des écoles (l'enseignement secondaire étant à l'époque fondé sur la séparation des genres) et les objectifs spécifiques de l'enseignement, les représentations de la femme véhiculées par les manuels d'enseignement du français, dans des situations diverses de la vie quotidienne (à la maison, dans la famille, entre amis, au travail, etc.), attestaient l'incompatibilité entre 
le modèle éducatif féminin et l'autonomie de l'analyse demandée par ce que nous nommons actuellement les "gender studies", qui intègrent le domaine des études francophones. La politique éducative féminine de l'«Estado Novo», promotrice de la femme au foyer (commebelle au bois dormant), prenait en suspicion tout désir d'émancipation de celle-ci. Simone de Beauvoir a pourtant été traduite en langue portugaise dans les années 1970.

Si nous comparons, d'autre part, les objectifs de cet enseignement avec ceux qui étaient assignés à l'enseignement du portugais, nous constatons que ces objectifs ne diffèrent pas du fait qu'ils s'appliquent à deux langues au statut spécifique: l'une, langue étrangère et l'autre, langue maternelle.

L'enseignement $d u$ français était alors bien révélateur, comme le constate Manuel Ribeiro pour "le discours pédagogique officiel» de l'époque, du «discours de légitimation culturelle et historique qui déformait la perception globale de la contemporanéité $\aleph^{38}$; à l'instar de l'enseignement du portugais, l'enseignement du français était bien révélateur d'une même "volonté de fusion inquestionnable avec certains "lieux de mémoire" glorifiants pour la Patrie " française dans ce contexte, d'après les lectures au programme de textes de la section «Images de l'Histoire de France », du manuel Regardons vers le pays de France, de Túlio Braz, $2^{\circ}$ ciclo. Ces lectures portaient sur les figures emblématiques que sont Saint Louis, Bayard, Louis XIV, Rouget de Lisle, le maréchal Lyautey, héros français au Maroc, Charlemagne ou Jeanne d'Arc, héros dont la lecture symbolique les rapprochait très clairement des héros nationaux.

Ceci nous autorise à nous demander quels étaient véritablement les objectifs de l'enseignement d'une langue étrangère à l'époque ? L'ouverture à l'autre, le besoin d'acquérir des outils de communication efficaces avec lui ? Ou bien, trouver dans ces enseignements la légitimation du bien-fondé d'une idéologie qui usaient de tous les moyens pour s'auto-promouvoir en toute occasion, tout en promouvant le caractère héroïque des figures nationales et de leurs faits?

L'énoncé du texte officiel du programme de français en vigueur entre $1955-1970^{39}$ est clair à ce propos :

L'explication de texte constituera l'exercice de base, la cible de la concentration mentale qui devra réguler les objectifs et orienter les activités. L'aspect verbal explication des mots - ne doit pas précéder le réel - l'explication des idées.

Tout est là : l'enseignement du français se devait d'être, avant tout, un enseignement pour les idées, ce qui revient à dire, un enseignement idéologiquement orienté ${ }^{40}$.

Quant aux finalités de l'enseignement secondaire ( $2^{\mathrm{e}}$ cycle, $10-15$ ans) :

L'étude de la langue française est intimement liée à l'effort commun de toutes les disciplines pour la formation, la culture et l'enrichissement de l'esprit. Les langues vivantes incluses dans le plan d'études du lycée sont l'expression de peuples desquels nous ne pouvons, en aucune façon, nous désintéresser, et, par conséquent, il importe que l'élève les lise, les traduise, les écrive et les parle avec assez d'aisance; que l'élève, en somme, les possède, dans la mesure où son âge et son développement mental le lui permettra.

Sur la « lecture expliquée et commentée » et sur les « exercices de traduction» :

En vérité, s'il est très utile de pouvoir traduire les pensées d'autrui d'une langue à l'autre, en faisant des exercices de version ou de traduction, il vaut beaucoup mieux avoir des idées à soi et les exprimer directement, et sans effort ni contorsion, dans la langue de l'autre peuple. Aux soins envers la correction et l'exactitude se joignent ici la réflexion, l'invention et l'élaboration formelle ${ }^{41}$. 

nes de ce cycle d'études) pour les classes débutantes, visaient en premier lieu, à contribuer à la "formation » de l'élève, plutôt que de lui «fournir des connaissances » pour les classes suivantes, cet enseignement devait d'abord être un «instrument de culture effectif ", à la recherche d' " une élégance sobre dans la conversation et dans les [textes] écrits $»^{42}$. Le texte du programme officiel ne manquait pas de signaler, dans ses normes pour les enseignements, qu'il fallait poursuivre des finalités d'ordre moral pour toute formation en langues étrangères, le législateur se montrant soucieux d'avertir les enseignants que les textes susceptibles de nuire à la bonne formation morale des jeunes seraient objet de censure. Ceci justifiait la politique du livre unique $»^{43}$ pour les manuels d'enseignement en vigueur pendant l'«Estado Novo", de même que l'importance des études de Lettres pour le régime, à l'époque, et, en particulier, des enseignements littéraires. Ceux-ci constituaient effectivement, un instrument privilégié, et je cite, pour la "formation intégrale " (à lire : utile) des élèves, dans l'ensemble de disciplines « unies » (à lire : unifiées) autour du culte des mêmes valeurs. Valeurs autour desquelles l'enseignement devait «mouler le caractère des élèves ». Ceci justifie que la pratique de l'analyse littéraire dans la discipline de français, bien qu'élaborée en français, se révèle également utile « au futur enseignant de portugais ou de français $»^{44}$.

72 C'est pourquoi apprendre le français constituait encore une excellente occasion pour apprendre à devenir portugais. Les représentations de la France et des Français transmises par l'enseignement du français étaient donc construites à partir d'un imaginaire national dont l'enseignement de l'Histoire ou de la Littérature portugaise s'évertuait à maintenir la flamme. et dans le raisonnement qui menait à l'adoption du "livre unique ", garant d'une formation «intégrale» des élèves. Celle-ci rassurait le régime devant le risque de la « ruine » de la nation, et du « chaos » et du « désordre » qui en adviendraient en cas de non-obédience à ces principes. Notions que l'avènement des études francophones allait miner de fond en comble ${ }^{45}$.

74 Aux craintes de la «ruine» du concept de langue/nation, les études francophones opposent le refus d'un rapport de possession à la langue ; aux craintes de «chaos » et de " désordre $»^{46}$, ces études répondent par la valorisation de nouveaux concepts, dont ceux d'hybridisme, de mixité, d'impureté, de mobilité, de transculturel.

Les principes "unifiants", monologiques et essentialistes de l'enseignement du français à l'époque se révèlent ainsi incompatibles avec le décentrement que présupposait l'émergence des études francophones. Ceux-ci privilégient la mobilité... la mondialisation de l'usage d'une langue, d'une langue en partage avec plusieurs cultures, avec d'autres cultures que celle d'origine. 

l'histoire (non hiérarchisé, non essentialiste) et à l'espace (sous le signe de la libre circulation entre les cultures et les modèles de comportement); de même qu'un rapport désacralisé à la langue, libérée de sa mission purement idéologique, et devenue outil de communication à des fins d'usages précaires, éphémères ou technologiques; à des usages «utiles » et non exclusivement " pour les valeurs », tel que l'enseignement de la langue à partir des textes littéraires " choisis » le préconisait, mais à l'existence d'autres valeurs, à d'autres modes d'expression.

77 L'émergence des études francophones s'est préparée de longue date au Portugal, au long d'un processus lent d'affranchissement du pays de son statut colonial. Un processus pendant lequel la France évoquait un pays d'accueil économique pour les uns mais aussi un mirage, un rêve de liberté pour d'autres. Au long d'un processus tout aussi lent d'affranchissement du pays de son statut de colonisé par des rêves de gloire que le régime nourrissait dès les bancs de l'école (dans les livres scolaires), par un imaginaire qui le projetait hors de soi (et où la France surgissait en premier lieu), faute de pouvoir s'y retrouver et s'y exprimer librement, l'espace de parole y étant usurpé par les discours patriotiques enflammés qui se refusaient à envisager d'autres solutions pour le problème africain, qui se refusaient à participer au concert des nations à l'ONU, par la résolution de la question coloniale ; un pays colonisé de l'intérieur par des voix rétrogrades et un aveuglement sans excuse. Un pays qui s'est retrouvé un jour et brusquement face à lui-même, sans "père ", honteux devant le processus inhumain d'une décolonisation abrupte et traumatisante, forcé à un choix : celui d'une Europe qu'il méconnaissait et qui le méconnaissait.

78 L'étude de l'émergence des études francophones constitue, à mon sens, un aspect particulier de la question plus générale des rapports entre le Portugal et la France, envisagés à différents moments de l'histoire des deux pays, et impliquant la planification de plusieurs niveaux de focalisation (pour employer une expression de l'analyse narratologique). Ceux-ci permettent le tissage de ponts entre plusieurs agents et facteurs qui s'impliquent les uns les autres.

79 Cette question gagne pourtant aussi à être contextualisée dans le cadre des rapports du Portugal avec lui-même.

Effectivement, les conditions de cette émergence se sont réunies lentement, au fil d'un processus identitaire douloureux pour ceux qui l'ont subi, et qui ont fait l'expérience de l'avant 1974 et de l'après cette date qui a marqué la rupture radicale entre deux visions du monde, entre deux manières d'être au monde, le Portugal "du Minho à Timor ", mais exclu du concert des nations libres. Les conditions de cette émergence suivent de près celle du Portugal "européen", dont l'équilibre lui permet désormais de se concevoir comme l'un des acteurs d'un espace culturel qui existe en dehors de ses frontières politiques - par le partage d'une langue et d'une histoire communes que cette appartenance demande à être revisitée. Cet espace est celui de la lusophonie, devenu possible lui aussi au bout de ce lent processus d'affranchissement de soi et de l'autre œuvré par l'Histoire.

81

Un processus d'affranchissement qui nous permet aujourd'hui d'en parler, d'en discuter librement, d'en faire des programmes d'enseignement et de recherche universitaire, des films pour le cinéma ou des documentaires pour la télévision, comme celui de Joaquim Furtado que la télévision portugaise diffusait, à la fin de l'année 2007 A Guerra -, sur la guerre coloniale et la politique portugaise de l'époque, d'en écrire des 
romans et des chroniques. Parmi ceux qui attirent le plus grand nombre de lecteurs, actuellement au Portugal, il convient de citer tout particulièrement les magnifiques textes d'António Lobo Antunes, jeune médecin et beau garçon, un de ces jeunes Portugais âgés d'une vingtaine d'années qui ont fait leur apprentissage de la vie, devant les corps déchiquetés de leurs camarades, blessés, estropiés par une mine ou tombés dans une attaque inattendue en Angola, en Guinée ou au Mozambique dans le courant des années soixante et soixante-dix du siècle dernier. Époque où j'apprenais, au lycée de jeunes filles, que ma patrie était la plus belle de toutes les patries et que je devais lui consacrer toutes mes pensées et même me sacrifier pour elle s'il le fallait...

Nous voilà plus de trente ans révolus après1974. C'est peut-être l'une des raisons pour lesquelles les auteurs francophones sont tranquillement (puisqu'ils n'éveillent plus de suspicion aujourd'hui) insérés au programme du secondaire et de l'université, non plus dans le but de respecter l'orthodoxie périodologique ou génologique d'une histoire de la littérature française à sens unique et obligatoire, reflet d'un régime désuet, mais parce que leur étude et la nature hybride de l'« indiscipline » francophone nous aide à mieux comprendre les enjeux de la contemporanéité, une contemporanéité marquée par les mouvances et les migrations transfrontalières, par les sonorités impures des langues miscigénées, par les transgressions des genres, dans la reconnaissance de l'appartenance du Portugal à cet ensemble de pays avec lesquels il partage une culture commune - marquée par toutes sortes de métissage -, en toute tranquillité, le Portugal étant devenu un pays comme les autres... ayant en partage avec bien d'autres encore une langue et des références historiques particulières, il est vrai,... tout comme la France et d'autres pays l'ont également...

\section{BIBLIOGRAPHIE}

MOSER, G., FERREIRA, M. (1983). Bibliografia das literaturas africanas de expressão portuguesa, Lisbonne, Imprensa Nacional-Casa da Moeda.

MOURA, J.-M. (1999). Littératures francophones et théorie post-coloniale, Paris, PUF.

MOURA, V. G. (2008). Acordo ortográfico : a perspectiva do desastre, Lisbonne, Alêtheia Editores.

\section{NOTES}

1. La bibliographie étant copieuse sur les fondements idéologiques du régime "Estado Novo ", je ferai uniquement référence aux discours de Salazar (Salazar, Discursos, Coimbra, Coimbra Editora, 1937-1967, 6 vols). Pour la dernière période du régime, 1968-1974, v. Caetano, Marcello, Depoimento, Rio de Janeiro/São Paulo, Distribuidora Record, 1974.

2. L'Ultimatum a provoqué des manifestations patriotiques du nord au sud du pays, pour la sauvegarde des territoires africains situés entre l'Angola et le Mozambique. L'hymne national portugais, créé pour l'occasion, et toujours en usage aujourd'hui, en est resté le signe le plus audible, vrai chant guerrier de rassem-blement national. 
3. Nous employons l'expression "études françaises" dans le contexte de l'ensei-gnement secondaire et universitaire, où elle relève de trois composantes fon-datrices : l'étude de la langue, de la civilisation et de la littérature.

4. Le mot "permanence » est sans doute le mieux adapté pour transmettre le maintien des programmes d'enseignement, lycéen ou universitaire, sans chan-gements significatifs au niveau des contenus d'enseignement en lettres, au long de plusieurs décennies, sous le régime de l'« Estado Novo ». L'un de ces facteurs est déterminé par le long espacement qui existe dans la chronologie des réformes de l'enseignement. Voir, à ce propos, les diverses publications de l'APHELLE (Associação Portuguesa para a História do Ensino das Línguas e Literaturas), depuis 2001, et Laurel, M. H. A., A história literária e o ensino da literatura francesa em Portugal (1957-1974), Universidade de Aveiro, 1989.

5. Globalement, nous pouvons considérer que la plupart des manuels scolaires en usage de nos jours poursuivent une orientation franco-française, sinon parisienne, et que l'ouverture aux francophonies y assume encore un caractère excentrique.

6. La connotation géopolitique de cette expression nous semble convenir à la situation décrite, en ce qu'elle conjugue les aspects territoriaux et la posture centra-lisatrice évoqués.

7. Jean-Marc Moura lance les fondements de la réflexion théorique, en langue française, sur les études postcoloniales francophones dans son livre, Littératures francophones et théorie postcoloniale, publié aux PUF, en 1999. V., en particulier, «Pour une théorie postcoloniale francophone », p. 139-160.

8. J.-M. Moura signale la disparition d'« Alioune Diop [1910-1980], l'un des fondateurs de Présence africaine [revue lancée simultanément à Paris et à Dakar en 1947, à laquelle collaborera Jean-Paul Sartre], de Paul Hazoumé [1890-1980], l'un des pionniers du roman négro-africain et de Boubou Hama [1906-1982], le penseur nigérien ", trois intellectuels dont la vie fut marquée par un engagement politique et littéraire influent. Pour la même période, l'auteur signale la «notoriété un peu biaisée de la littérature maghrébine [...] liée à un intérêt français pour l'actualité qui négligeait sa littérarité », le manque de dynamisme des littératures de «l'océan indien, du Pacifique et de l'Indochine ", et "l'émergence d'une nouvelle 'esthétique du plurilinguisme' à partir de 1980 », dans la littérature québécoise (Moura, J.-M., 1999 : 139-140).

9. Pour une vision d'ensemble de ces littératures, consulter C. Bonn et al. (dir.), La littérature maghrébine de langue française, Paris, EDICEF-AUPELF, 1996 ; J. Chevrier, Littératures d'Afrique noire de langue française, Paris, Nathan, 1999 ; id., Littératures francophones d'Afrique noire, Aix-enProvence, Edisud, 2006 ; L. H. Hoffmann, Bibliographie des études littéraires haïtiennes, 1804-1984, Vanves, EDICEF-AUPELF, 1992 ; Ngandu, P. H., Écritures littéraires: dictionnaire critique des ceuvres africaines de langue française, La Nouvelle-Orléans, Presses Universitaires du Nouveau Monde, 2002, 2 vols.

10. V. Salema, Maria José, A didáctica das línguas vivas e o ensino do francês nos liceus portugueses na viragem do século: o período de 1894 a 1910, Braga, Universidade do Minho, thèse de doctorat dactylographiée. L'année même où le roi des Belges, Léopold I ${ }^{\mathrm{er}}$, appuie la monarchie portugaise, dans ses prétentions à la province du «Congo », située au Nord de l'Angola, plus tard incorporée au Congo belge.

11. V. O ensino particular das línguas e literaturas estrangeiras em Portugal, Actas das Primeiras Jornadas APHELLE, Lisboa, Universidade Católica Portuguesa, 8 de Março de 2002.

12. Maria José Salema s'est occupée de l'enseignement religieux privé (fin XIX ${ }^{\mathrm{e}}$ ) et de l'enseignement militaire (1900-1936) dans une institution pour des jeunes filles, filles de militaires dans «O ensino das línguas estrangeiras no Instituto de Odivelas entre 1900 e 1936 », Actas do III Colóquio APHELLE : Para uma história do ensino das línguas e literaturas estrangeiras : estudos de género, Universidade do Algarve, 23-24 de Junho de 2005 : 139-154.

13. Marcel Proust avait été le lauréat du prix Goncourt deux années auparavant. 
14. Par Aimé Césaire, au nº 3 de la revue, en 1935. L. S. Senghor l'emploiera plus tard également, dans son recueil poétique,Chants d'Ombre (1945).

15. A. Diop, président de l'Assemblée, et L. S. Senghor, président de la République, étaient alors en charge des postes politiques les plus marquants dans leur pays.

16. Decreto-Lei no 35 046, du 22 octobre.

17. Le numéro de novembre 1945 de la revue Vértice lance un appel à la réali-sation d'élections libres, à l'institution de partis politiques, à un nouveau recen-sement, bref, à la prise de conscience de la situation et à l'action d'une "forte opposition démocratique ", tel qu'on peut lire dans son éditorial, intitulé « Le pays et les élections ».

18. Le Brésil est la première colonie devenue indépendante, en 1829 , d'une manière pacifique.

19. Les travaux commencés en 1910 par des linguistes portugais éminents, dont G. Viana, A. dos Reis, C. Michaëlis et C. Figueiredo, qui proposent des changements orthographiques profonds, tels l'abolition du « $\mathrm{y}$ » ou des doubles consonnes «ph », «ll», «th», ne seront pas extensifs au Brésil. Les travaux de l'Académie des Sciences de Lisbonne et de l'Académie brésilienne des Lettres ont débuté en 1924. Sept ans révolus, le premier accord orthographique est approuvé entre les deux pays, sans qu'il ait été pourtant jamais appliqué. L'histoire de cet accord se poursuivra jusqu'à la célébration de la Première Convention Orthogra-phique entre le Portugal et le Brésil en 1943.

20. La « résistance » du Brésil tout au long de l'histoire de cet accord est tout aussi significative d'une histoire postcoloniale commune, ponctuée d'écarts et de rapprochements.

21. Il est sans doute tout aussi significatif que ce soit le Brésil qui ait promu, en 1986, donc, bien au-delà de la période qui nous occupe, la première rencontre des communautés de langue officielle portugaise, justement, celle des anciennes colonies portugaises.

22. La ratification, toute récente, par le Conseil des ministres, le 6 mars 2008, d'un accord dont les bases étaient établies dès 1990 par le consensus de la Communauté des pays de langue portugaise (CPLP), et qui privilégie nettement la graphie brésilienne, aux dépens de la graphie portugaise a suscité au Portugal la plus vive polémique, opposant des figures de référence de l'intellectualité portugaise dont, celles de quelques universitaires comme Carlos Reis, Maria Alzira Seixo ou Vasco Graça Moura. Ce dernier vient de publier une synthèse des arguments qui soutiennent le refus de l'accord, in Acordo ortográfico : a perspectiva do desastre, Lisbonne, Alêtheia, 2008. («Accord orthographique : la perspective du désastre »). La graphie portugaise constitue la norme de quelque 40 millions d'utilisateurs, ce nombre comprenant les utilisateurs de la langue au Portugal et ceux des pays de langue portugaise, de même que les communautés émigrantes de la diaspora portugaise dans le monde, contre les quelque 186 millions d'utilisateurs brésiliens.

23. L'histoire de l'Alliance française est révélatrice de cette option. V. Bruézière, Maurice, L'Alliance française : histoire d'une institution, Paris, Hachette, 1983.

24. Sur les rapports entre l'idéologie politique, le régime juridique et les pratiques d'enseignement du français au Portugal dans les années soixante au Portugal, v. Garcia, M. G. D. et Laurel, M. H. A., «Ensinar francês tem género ? Breve reflexão sobre o discurso jurídicopolítico e os livros de ensino dos anos sessenta", in Para uma história do ensino das línguas e literaturas estrangeiras : estudos de género, Actas do III Colóquio APHELLE, Universidade do Algarve, 2007 : 213-256 ISBN 972-99682-1-7.

25. Eduardo Lourenço s'interroge sur la permanence de ce sentiment dans la notion moderne de "lusophonie », en particulier dans A nau de Ícaro - Imagem e miragem da lusofonia, Lisbonne, Gradiva, 1999, et Situação Africana e Consciência Nacional, essai que l'auteur ne pourrait publier qu'en 1976, deux ans après le changement du régime politique, mais dont la genèse remonte à son séjour au Brésil, entre 1959 et 1960, et dont la thématique inspire 0 Labirinto da saudade. Nous remercions notre collègue Maria Manuel Baptista, spécialiste de Eduardo Lourenço, pour ces informations. Sur le luso-tropicalisme, v. J. B. de Macedo, «O luso-tropicalismo de Gilberto Freire ", 1989, ICALP, vol. $15: 131-156$. 
Moisés de Lemos Martins, in «Lusofonia e luso-tropicalismo : equívocos e possibilidade de dois conceitos hiper-identitários », s'interroge sur « l'équivoque luso-centré » éventuellement sousjacent aux deux concepts de "lusophonie» et "luso-tropicalisme». Ce dernier, formulé par l'essayiste brésilien Gilberto Freire, a inspiré l'idéologie de l'« Estado Novo » dans le domaine des relations extérieureset dans celui de la politique de la langue.

26. Pour certains, ce choix aurait constitué une stratégie diplomatique conçue dans le but d'éviter, en quelque sorte, l'article 73 de la Charte de l'ONU qui obligeait les puissances coloniales à informer l'ONU sur le processus des autonomies.

27. Decreto-Lei (décret-loi) no 39749 , du 9 août.

28. «Orgulhosamente sós », en portugais. Cette expression a été prononcée par Salazar en 1965, lors d'un discours proclamé en faveur du maintien de la guerre coloniale et des valeurs de la civilisation portugaise (par métonymie, occidentale), en Afrique (v. Franco Nogueira, Salazar, vol. VI, O último combate (1964-1970), Porto, Livraria Civilização, 1985 : 7-8). L'expression a aussitôt été interprétée comme la marque de la politique extérieure portugaise. A partir des années 1990, de nombreux historiens proposent une interprétation plus approfondie de cette période du régime, en accentuant l'appui implicite accordé par la France de De Gaulle et l'Allemagne d'Adenauer et de Strauss, entre autres pays, dont l'Afrique du Sud, à la politique coloniale portugaise. Parmi ces historiens, nous citons les noms de Fernando Rosas, Norrie Macqueen, António José Telo, António Costa Pinto.

29. «Estado português da Índia » (l'État portugais de l'Inde), formé dès le XVI ${ }^{\mathrm{e}}$ siècle, par Goa, Daman et Diu.

30. Zita Seabra, dissidente du parti communiste portugais, en fait le constat dans un livre publié en 2007, Foi assim (éd. Civilização). Agostinho Neto, premier président de la république angolaise, fut un de ces étudiants, à Moscou.

31. Cette courte période fut appelée le «printemps marcelliste», par similitude avec le «printemps de Prague».

32. Gerald Moser e Manuel Ferreira, Bibliografia das literaturas africanas de expressão portuguesa, Lisbonne, Imprensa Nacional-Casa da Moeda, 1983.

33. Il s'agit de la réforme du ministère de Carneiro Pacheco. La désignation de ministère de l'instruction publique (fondé lors de la I ${ }^{\text {ère }}$ République), est alors remplacée par celle de ministère de l'éducation nationale, beaucoup plus expressive du point de vue idéologique. L'éducation, qui devrait mettre l'accent sur les principes davantage que sur les contenus; nationale: une éducation tournée vers l'intérieur, vers la sauvegarde des valeurs identitaires de la nation. Le sentiment d'orgueil de la race, de la Patrie et de leurs valeurs civilisatrices/missionnaires se trouve condensé dans cette nouvelle désignation, que la fête nationale du 10 juin consacrait. Le fondement élitiste de la culture prôné par cette réforme correspond à la fondation, la même année, de l'Institut pour la haute culture (Instituto de Alta Cultura) : 1936-1977.

34. Roman dont la première traduction en langue portugaise, Admirável Mundo Novo, par Mário Henrique Leiria, [1955], a été faite par Livros do Brasil, maison d'édition fondée en 1944, précédée d'une traduction brésilienne de 1941. Le Meilleur des mondes est le titre de la traduction française. 35. À cette date, la structure des études d'humanités était encore proche de celle des réformes de 1918 et de 1930.

36. Aucune thèse en études françaises ne fut soutenue au Portugal entre 1945 et 1970. La première fut soutenue en 1977 à l'université de Lisbonne, par Maria Alzira Seixo, la doyenne de ces études au Portugal : Le parcours du plaisir. Essai d'analyse d'un texte français du XVII ${ }^{e}$ siècle: "Francion", de Charles Sorel, publiée en 1985 à Paris par la Fondation C. Gulbenkian, thèse dirigée par Roland Barthes (EPHE) et Jean Rousset, professeur à l'université de Genève. Ce travail inaugure l'autonomie relative des études françaises face aux études portugaises à l'université.

37. V. l'article cité dans la note de bas de page, no 24 . Nous traduisons tous les passages cités de cet article. 
38. Ribeiro, Manuel, « $O$ discurso pedagógico oficial e a literatura: questões de identidade cultural e histórica », Vértice, 65, 1995 : 71-79. Notre traduction.

39. Decreto o 39 807, publié dans le Journal officiel du gouvernement (Diário do Governo) du 7 septembre 1954. Nous traduisons les passages cités de cette loi.

40. V. article cité, note 24, p. 232 (note 1) et p. 235.

41. Article cité, note 24, p. 233.

42. Ibid., p. 234.

43. Décidé lors de la réforme de Carneiro Pacheco, 1936. Cf. Article cité, note 24, p. 237.

44. Article cité, note 24, p. 234 et p. 235.

45. Ces deux « dangers » sont encore évoqués par les partisans de l'exclusivité des études francofrançaises à l'université portugaise, dans leurs arguments ana-chroniques contre les études francophones, qui ne sont pas loin d'évoquer la mentalité nationaliste et coloniale de l'« Estado Novo ». Le décentrement sous-jacent aux études francophones implique l'annulation de la triade nation/langue/culture; le renforcement des perspectives comparatistes; le refus des perspectives essentialistes, chronologiques et exhaustives de l'étude de la littérature.

46. Cf. Salazar, op. cit., Préface à Discursos, 1935, vol. III.

\section{RÉSUMÉS}

Malgré le succès de la diffusion du concept de «francophonie » à partir des années soixante du siècle dernier, en France et dans l'ensemble des anciens pays colonisés, il faudra reconnaître que ce succès ne fut pas partagé, à part égale et en même temps, par tous les pays où le français était objet d'enseignement et véhicule de transmission culturelle. L'histoire de l'enseignement du français porte l'empreinte des circonstances idéologiques et politiques qui caractérisent l'histoire des pays où cette pratique a lieu; tel est le cas, en l'occurrence, du Portugal, où la période comprise entre 1945 et 1970 s'insère en pleine période coloniale de son histoire, les années soixante et soixante-dix ayant constitué des années décisives de ce cycle.

Nous nous proposons de considérer les conditions de l'impossibilité de l'émergence du concept de francophonie, au sens moderne du mot, au Portugal pendant cette période, ce concept dénotant des risques considérables pour le régime. En tenant compte de la présence et du rôle des institutions officielles françaises sur le terrain pendant cette période (Institut français, Alliance française), des programmes d'enseignement suivis dans les lycées, et des tendances vérifiées dans les études françaises à l'université, nous nous interrogerons surtout sur l'adéquation de certains choix pédagogiques au maintien du régime et de ses options idéologiques, pour conclure (très rapidement, cette période dépassant les dates prévues pour ce colloque) que ce ne sera qu'à partir de la dernière décennie du $\mathrm{XX}^{\mathrm{e}}$ siècle que le concept de francophonie portera ses fruits au Portugal, plus de vingt ans après la décolonisation, suivant de près l'émergence des études lusophones, notamment à l'université.

Notwithstanding the success achieved in the diffusion of the "francophone" concept in the past century during the sixties in France, and in the group of nations colonized by it in the past, we have to admit that this success was not shared in the same way nor at the same time, by all those countries where French was taught and considered a cultural promotional vehicle. Historically speaking, French teaching is marked by political and ideological circumstances which characterize the history of the countries in which this practice took place; such is the case with 
Portugal, a country where the period between 1945 and 1970 is in full colonial period of its history, being the sixties and the seventies the most decisive years in this cycle.

We would like to consider the conditions which made it impossible for the "francophone" concept to rise, in the modern sense of the word, in Portugal during this period, a concept which meant added risks for the regime. Considering the presence and role of official French institutions on the terrain during this period of study (Institut français and Alliance française), of the syllabi being followed at State Schools, and the tendencies of French studies at universities, we question ourselves, above all, on the suitability of some of the pedagogical choices, in maintaining the regime and its ideological options, to conclude (very briefly, due to the fact that this period exceeds the dates foreseen for this debate) that only in the last decade of the 20th century will the concept of "francophone" bear fruit in Portugal, more than 20 years after colonization ended, closely following the rise of Portuguese studies at university level

\section{INDEX}

Keywords : African magazines in Portuguese, Estado Novo, Portuguese colonies, representations of France and Francophonie, teaching languages and literature policies

Mots-clés : colonies portugaises, Estado Novo, politiques d'enseignement des langues et des littératures, représentations de la France et francophonie, revues africaines en langue portugaise

\section{AUTEUR}

MARIA HERMÍNIA AMADO LAUREL

Université de Aveiro, Portugal

hlaurel@ua.pt 